\title{
Investigation of a turbulent spot and a tripped turbulent boundary layer flow using time-resolved tomographic PIV
}

\author{
Andreas Schröder · Reinhard Geisler • \\ Gerrit E. Elsinga $\cdot$ Fulvio Scarano . \\ Uwe Dierksheide
}

Received: 30 September 2006/Revised: 7 September 2007/ Accepted: 17 September 2007/Published online: 13 October 2007

(C) Springer-Verlag 2007

\begin{abstract}
In this feasibility study the tomographic PIV technique has been applied to time resolved PIV recordings for the study of the growth of a turbulent spot in a laminar flat plate boundary layer and to visualize the topology of coherent flow structures within a tripped turbulent flat plate boundary layer flow. The experiments are performed around $\left(R e_{x}\right)^{1 / 2} \approx 450$ in a low speed wind-tunnel using four high speed CMOS cameras operating up to $5 \mathrm{kHz}$. The volume illumination required a multiple-reflection system able to intensify light intensity within the measurement volume. This aspect is deemed essential when a high-speed tomographic PIV system is applied in air flows. The particle image recordings are used for a three dimensional tomo-
\end{abstract}

This research article was submitted as a selected contribution from the 12th international symposium on application of laser techniques to fluid mechanics, June 26-29, 2006.

\footnotetext{
A. Schröder $(\bowtie) \cdot$ R. Geisler

Deutsches Zentrum für Luft- und Raumfahrt,

Institut f. Aerodynamik und Strömungstechnik,

Bunsenstrasse 10, 37073 Göttingen, Germany

e-mail: andreas.schroeder@dlr.de

R. Geisler

e-mail: reinhard.geisler@dlr.de

G. E. Elsinga $\cdot$ F. Scarano

Department of Aerospace Engineering,

Delft University of Technology, Delft, The Netherlands

e-mail: g.e.elsinga@tudelft.nl

F. Scarano

e-mail: f.scarano@tudelft.nl

U. Dierksheide

La Vision GmbH, Anna-Vandenhoeck-Ring 19,

37081 Göttingen, Germany

e-mail: udierksheide@lavision.de
}

graphic reconstruction of the light intensity distribution within the illuminated volume. Each pair of reconstructed three-dimensional light distributions is analyzed by 3D spatial cross-correlation using iterative multi-grid schemes with volume-deformation, yielding a correlated time sequence of three-dimensional instantaneous velocity vector volumes. The coherent structures organization is analyzed by 3D-vorticity and -swirling-strength iso-surfaces visualization. In both flow types streaks and hairpin-like or arch vortical structures are most prominent. The data gives insight into the role of these structures for the spatiotemporal arrangement of the wall normal flow exchange mechanisms, especially of the instantaneous Reynolds stress events Q2 and Q4. A description of different self-sustainable flow organizations based on modifications of the hairpinvortex- and streak-models is given. Two preliminary results are essential: Self-sustainability of a coherent vortical structure depends on the ability to entrain high momentum fluid, initially Q4. And, stream-wise swirl at the near-wall region of arch or hairpin-like vortices has been observed to be rare.

$\begin{array}{ll}\text { List of symbols } & \\ t & \text { time } \\ V x, V y, V z & \begin{array}{l}\text { instantaneous velocity components in } x \text {-, } \\ y \text {-and } z \text {-direction } \\ \text { fluctuation velocity components }\end{array} \\ V^{\prime} x, V^{\prime} y, V^{\prime} z & \begin{array}{l}\text { streamwise, normal and spanwise } \\ \text { coordinates }\end{array} \\ R, y, z & \begin{array}{l}\text { Reynolds number based on length from } \\ \text { plate LE }\end{array} \\ R_{x} & \text { time-difference from excitation of spot } \\ T & \text { focal length }\end{array}$




$\begin{array}{ll}f_{\#} & \text { aperture stop } \\ Q 1, \ldots, Q 4 & \begin{array}{l}\text { Quadrants of instantaneous Reynolds } \\ \text { stress } V^{\prime} x V^{\prime} y\end{array} \\ U_{\infty} & \text { free-stream velocity } \\ \delta & \text { boundary layer thickness }\left(0.99 U_{\infty}\right) \\ \lambda & \text { wave length of laser light } \\ \text { |rotl } & \text { magnitude of instantaneous vorticity }\end{array}$

Abbreviations

PDF

RMS

(T)BL

TE

LE

TR

\section{Introduction}

Turbulent spots are small patches of turbulent flow which appear naturally within laminar boundary layer (BL) flows around and beyond the critical Reynolds number. These spots are often initiated by non-linear disturbances of the laminar flow at a local position and are growing downstream in stream- and span-wise directions. A turbulent spot is a special bypass transition scenario. The disturbance develops into an area of turbulent flow in a self-similar arrowhead shape convecting downstream of the disturbance event (Fig. 1).

When several spots at different span-wise locations merge, the flow develops into a turbulent boundary-layer, Emmons (1951). Previous research on artificially generated turbulent spots utilized mostly point measurement methods and visualization techniques, while the latter returns the model of hairpin structures evolving at the trailing edge of the spot, Matsui (1980), and wavy streaks close to the wall, Gad-el-Hak et al. (1981). Models of unsteady events of the

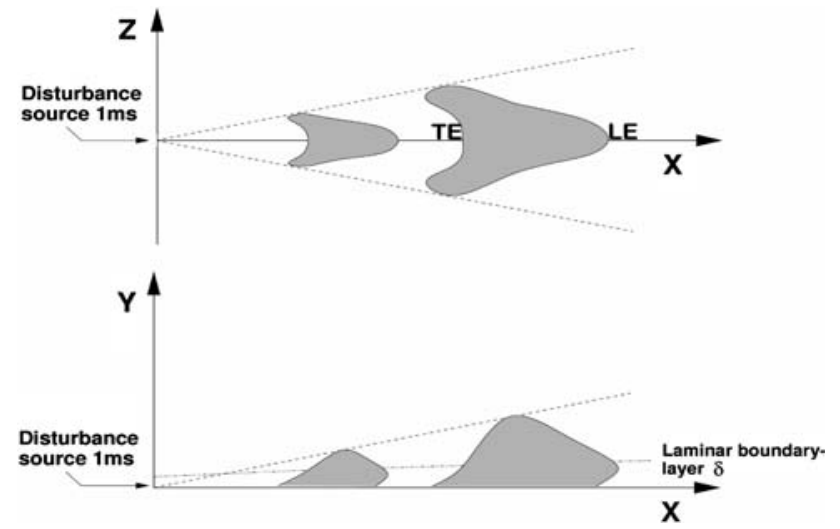

Fig. 1 Development of a turbulent spot in a laminar boundary layer (grey $>$ threshold rms-value) coherent substructures based on hot wire anemometry, like sweeps and ejections, required the application of Taylor's hypothesis. Some basic features of the spot have been measured and described as there are spreading angle, shape, altitude, growth rates and linear instability events in the vicinity of the spot, Cantwell et al. (1977), Wygnanski et al. (1982). Other results were achieved by conditional averaging, which gave valuable quantities of the overall structure and also of the convection velocity and inclination angle (about $45^{\circ}$ ) of the substructures, Sankaran et al. (1987). It has been concluded that the growth characteristics and shape of the spot in flat-plate BL flows are depending on the Reynolds number, pressure gradient, surface roughness, free-stream turbulence level and disturbance amplitude and wavelength (Gostelow et al. 1996). Recent numerical simulations of turbulent spots and of cascades of evolving hairpin vortices reached a considerable accuracy and spatial resolution, in substantial agreement with experimental observations (Singer 1996; Tufo et al. 1999). The substructures of the turbulent spot seem to remain self-similar in all cases and have been recognized as low- and high speed streaks and hairpin-like vortices very similar to those appearing in fully developed turbulent BLs but more orderly (Schröder und Kompenhans 2004). A similar behaviour has been reported applying the time-resolved Stereo PIV technique to turbulent spots (viz. puffs), in a pipe flow (Doorne van et al. 2003), where the ensemble of substructures also consists of a limited number of streaks near the wall and hairpin-like vortices organizing the turbulent flow exchange. More quantitative information about the instantaneous topology of the substructures within the spot as the main carriers of the turbulent mixing process is still desired. Some aspects of this process were understood by the help of experiments performed with PIV in conjunction with liquid crystal thermography (Sabatino and Smith 2002), providing additional insight into the heat transfer characteristics of the spot associated to the local topology of the substructures.

Summarizing the main results obtained so far: the turbulent spot is arrowhead-shaped with the tip pointing downstream (Fig. 1) and its thickness develops similarly to that of a turbulent BL. Hairpin-like vortices evolve at the trailing-edge (TE) and increase the size of the turbulent spot in stream- and span-wise direction. Their inclination is about $45^{\circ}$ to the wall and they convect downstream with approximately $75 \% \mathrm{U}_{\infty}$ depending on the distance from the wall. The average TE convection velocity is ca. $62 \% \mathrm{U}_{\infty}$ and the leading-edge (LE) velocity ca. $87 \% \mathrm{U}_{\infty}$. An overhang region characterizes the LE of the spot consisting of the convecting, dissipating and stretched vortex heads above a laminar layer. Typically stream-wise streaks appear in the near-wall region and persist behind the TE of the spot. A region of higher velocity, the s.c. "calmed 
region", with an adverse pressure gradient (Coles and Savas 1980) is following the spot. The turbulent mixing inside the spot due to the (instantaneous) negative Reynolds stresses (the so called Q-2- and Q-4-events), is connected to a staggered pattern of hairpin-like vortices, according to the idealized mixer-model of trailing-edge substructure formation of the spot proposed by Schröder and Kompenhans (2004). In a previous study Schröder (2001) proposed a model generated out of an interpretation of the spatial distributions of space-time correlations of the single Reynolds stress elements calculated for different planes parallel to the wall. Following that model the initial process for the (re-)formation of hairpin-like vortices seems to be a Q4-event which was deflected by a downstream flow structure and transformed into a Q3-event, which then interacts with a wall-near low speed streak resulting in the rapid production of a Q2 event. This assumption has to be proven with a fully $3 \mathrm{D}-3 \mathrm{C}$ and time resolved measurement technique. The turbulent spot consists of a pattern of complex hairpin-like substructures which develop explosively when the "calmed" laminar flow upstream reaches the trailing-edge of the spot. Inside the spot these substructures interact and govern the turbulent mixing process.

In the past two decades considerable experimental and numerical work on coherent structures in wall bounded turbulent flows has been carried out. A focus for a direct comparison of the results of the present work will be the spatio-temporal models of coherent flow structures in such flows, which have been developed partly by using the PIV techniques (e.g. Robinson 1991; Meinhart 1994; Schoppa and Hussain 1997; Tomkins et al. 2003; Kähler 2004). Since almost two decades the PIV technique has enabled the determination of instantaneous velocity vector fields in a plane of the flow, returning important results based on instantaneous and statistical approaches to the analysis of the turbulent flow structures. Successful extensions of PIV experiments towards dual plane and/or time resolution have been performed during that time and their applications to turbulent flows emphasized the role of coherent flow structures for this research field.

A recent application of the tomographic PIV technique to a tripped turbulent boundary layer flow at a low Reynolds number has been reported by Elsinga et al. (2007). In this paper instantaneous spatial topologies of the coherent structures like hairpin vortices, packets of hairpins along low speed streaks and Q2-events have been visualized segmented in 3D quantitatively with a very high quality. The coherent structures have been extensively described and global statistics have been calculated for comparison with theory and literature. However, the desired step towards a fully three-dimensional and time-resolved extension of PIV for the investigation of turbulent wall bounded flows has been up to now mainly hampered by technical restrictions. The first time preliminary results of time-resolved 3D-3C velocity vector fields from turbulent wall bounded flows gained by using tomographic PIV have been published by Schröder et al. (2006).

The present investigation adopts the tomographic PIV technique with a high-repetition rate illumination-recording system, allowing performing a quantitative threedimensional and time-resolved velocity measurement of two types of wall bounded turbulent flows: turbulent spots in a laminar BL and a tripped turbulent BL flow. The work contribution focuses on giving insights into the temporal evolution of the 3D flow organization and the exchange mechanisms governed by coherent structures inside these two turbulent flow types. In particular, the rapid formation process of hairpin-like vortices at the trailing-edge of the spot is scrutinized. The described flow structure topologies and characteristics in a turbulent spot are quite similar to those in fully developed turbulent BL flows. For a better comparison of the classical coherent structure models it is of advantage to mimic the fully developed turbulent BL flow closer by a tripped turbulent BL flow case, which was realized by span-wise tripping wires. In that case the coherent structures appear homogeneously along the spanwise and stream-wise directions, therefore enabling a thorough statistical description of the coherent structures.

The present measurements demonstrate that the degree of complexity of the flow structures, more or less expected for a $3 \mathrm{D}-3 \mathrm{C}(t)$ representation of instantaneous velocity vector volumes, suggest the formulation of a modified topological organization of the flow in comparison with existing models of coherent structures. The work concludes with the comparison between the local and global by-pass transition scenario. Beside time series of instantaneous 3D velocity vector fields, 3D-vorticity and swirling strength fields are represented by iso-surfaces, which highlight the topology of individual vortical substructures, their time development and induced flow. Swirling strength is the imaginary part of the locally calculated complex eigenvalue of the velocity gradient tensor, which is a measure for rotation excluding shear vorticity (Zhou et al. 1999).

\section{Experimental apparatus and procedure}

\subsection{Flow facility}

The laminar flat plate boundary layer flow was generated at the open test section of the circuit type $1 \mathrm{~m}$-wind tunnel of the DLR, Göttingen, at a free stream velocity of $U_{\infty}=7 \mathrm{~m} / \mathrm{s}$ with zero pressure gradient. A transparent and very smooth surface glass plate is utilized to provide optical access without distortions from its bottom and a 
good flow quality. The plate has an elliptic leading edge to prevent flow separation. With a short $\sim 1.5 \mathrm{~ms}$ local flow injection at $\left(R e_{x}\right)^{1 / 2}=300$ an intense initial disturbance has been introduced into the laminar boundary layer flow. The response of a $6 \mu$ s long TTL input signal after amplification is shown in Fig. 2. This voltage peak signal with $\sim 1.5 \mathrm{~ms}$ duration fed a pressure chamber loudspeaker $(2 \times 60 \mathrm{~W})$ which produces a short pressure wave passing via a plastic tube to the disturbance source. Flow direction is defined as $x$-axis, wall normal direction as $y$-axis and span-wise direction as $z$-axis. The flow injection through a slit of $0.5 \times 2 \mathrm{~mm}^{2}$ in $x-z$-direction is perpendicular to the surface and initializes flow structures which lead to the formation of a turbulent spot. Downstream of this local disturbance the spot grows in span- and stream-wise directions while convecting downstream as depicted in sketch of Fig. 1. After a time difference of $\mathrm{T} \approx 70 \mathrm{~ms}$ after the flow injection the $\mathrm{LE}$ and after $\mathrm{T} \approx 90 \mathrm{~ms}$ the $\mathrm{TE}$ of the spot passes through the measurement volume, which was a box of about $32 \times 18 \times 29 \mathrm{~mm}^{3}$ in $x-y$ and $z$ directions around $\left(R e_{x}\right)^{1 / 2} \approx 450$ illuminated by laser light pulses at $5 \mathrm{kHz}$ in order to achieve PIV recordings without using the frame straddling method. The evaluation by volume cross-correlation result in a time series of instantaneous velocity vectors volumes with $200 \mu$ s step size.

Secondly an analogous experiment with a wire-tripped flat plate turbulent boundary layer flow has been performed in order to reveal the differences of both flow types. Four tripping wires, one with $1.5 \mathrm{~mm}$ diameter and three with $1 \mathrm{~mm}$ diameter were positioned with $1 \mathrm{~mm} x$-distance close to the surface at $\left(R e_{x}\right)^{1 / 2} \approx 290$ along the whole span-wise elongation of the plate. This bypass transition or tripping of the flow results in the development of a turbulent boundary layer flow, which has been measured in a volume box of about $34 \times 18 \times 35 \mathrm{~mm}^{3}$ at the same distance from the plate's leading edge as for the spot case. The frame- and laser pulse rate was $4 \mathrm{kHz}$ and the cross-correlation of the reconstructed particle image volumes has been calculated without using frame straddling that means instantaneous velocity vector volumes representing time steps of $250 \mu$ s each.

\subsection{Measurement technique}

High-repetition rate PIV system has recently appeared with technical specifications enabling to operate PIV in timeresolved mode for low speed air flows. There is, however, a compromise between the repetition rate and the available laser pulse energy. Typically lasers pulse energy at $1 \mathrm{kHz}$ is about one order of magnitude less than that for low reprate systems. Moreover high rep-rate CMOS sensors are lower quality imagers compared with CCD cameras, but light sensitivity of the CMOS technique increases significantly the past years.

The tomographic technique poses even higher requirements in terms of illumination intensity and spatial resolution of the imaging system because the measurement volume is typically one order of magnitude larger than that for planar PIV. Moreover, the imaging system requires a finite depth of focus corresponding to the thickness of the illuminated region. This is usually achieved at the cost of a smaller optical aperture of the objectives, causing a further decrease of the particle image intensity.

\subsubsection{Illumination system}

The illumination source is a dual-cavity diode pumped Nd:YAG laser from Lee Laser Inc. with a total pulse energy of $21 \mathrm{~mJ}$ per pulse at $5 \mathrm{kHz}$ (pulse duration $80 \mathrm{~ns}$ at $532 \mathrm{~nm}$ ). For the tripped boundary layer the laser is operated at $4 \mathrm{kHz}$ at the same pulse energy. The laser beam passes through a beam expander and collimating optics and is then directed towards the measurement region parallel to the wall.

Two coated and highly reflective dielectric mirrors of $50 \times 30 \mathrm{~mm}^{2}$ aligned in the $x$-y-direction are installed
Fig. 2 Photo of a digital oscilloscope display with inverse trigger signal input (bottom line) and amplifier output (top line) (left) for introducing a initial disturbance of the laminar BL flow by a vertical flow injection through a flush mounted slit downstream of the elliptical leading edge (right)
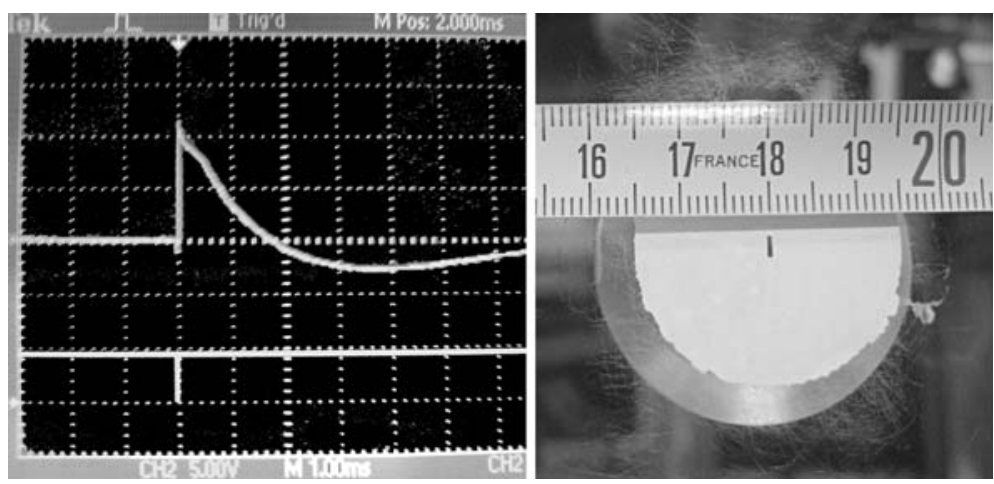
nearly parallel and close to the surface of the flat plate on opposite sides of the measurement region. The collimated laser beam of about $20 \mathrm{~mm}$ diameter in cross section was directed almost normal to one mirror. The beam undergoes multiple reflections between the two mirrors passing through the measurement volume approximately 15 times before exiting (see sketch in Fig. 3). This arrangement resulted in a light amplification with a factor $\sim 5$ with respect to the case of a single pass system. The lower limit of the illuminated volume was realized by the lower mirror edge parallel to the flat plate at $y=1 \mathrm{~mm}$. For a straight upper cut of the light volume an appropriate faceplate has been mounted parallel to the plate at $y=20 \mathrm{~mm}$. The resulting illuminated region was $35 \times 19 \times 1200 \mathrm{~mm}^{3}$.

\subsubsection{Imaging and recording}

The scattered light from the illuminated seeding particles is recorded by four cameras placed along an angle of $\sim 40^{\circ}$ in between two cameras of each lateral side and $\sim 35^{\circ}$ towards the flat plate. The specific illumination arrangement has the additional advantage that all viewing directions collect scattered light both in forward and backward direction, which provides the magnitude of light needed for imaging particles according to Mie-theory.

Four Photron APX-RS CMOS cameras $(1,024 \times 1,024$ pixels at $3 \mathrm{kHz}$ ) are equipped with Nikon lenses. The lenses used had a focal length $f=100 \mathrm{~mm}$ and an aperture of $f_{\#}=8$, yielding a focal depth of approximately $12 \mathrm{~mm}$. The Scheimpflug condition between the image plane, lens plane and the mid-object-plane (see Fig. 4) is obtained with free-rotating tilt adapters. As a result the particles at

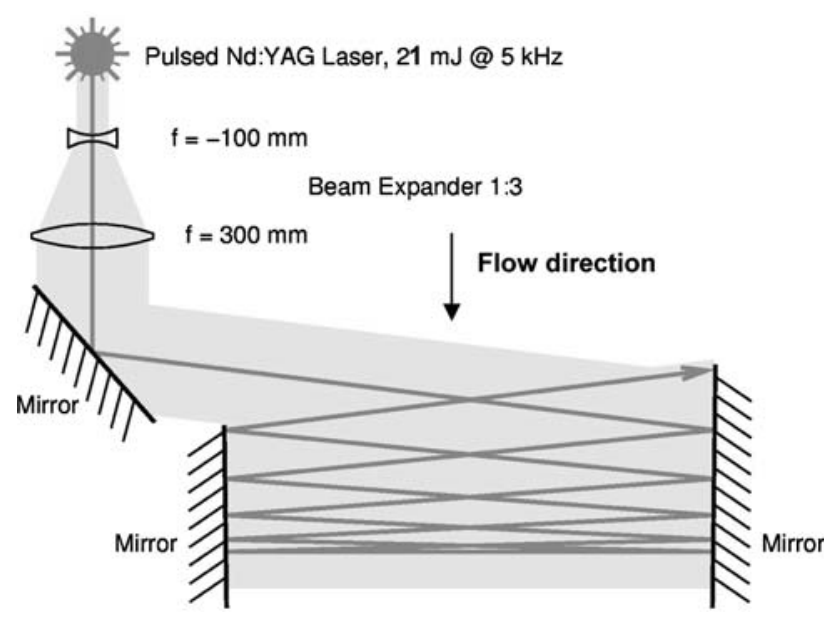

Fig. 3 Sketch of the optical setup which enables the optimal usage of the laser pulse energy of only $21 \mathrm{~mJ}$ per pulse for illumination of the PIV measurement volume at 4 and $5 \mathrm{kHz}$ the edges of the measurement volume (in depth) are slightly blurred; however this does not severely affect the tomographic reconstruction, in that particle images have to overlap several camera pixels anyway. In addition the camera pixels size is rather large $\left(17 \times 17 \mu \mathrm{m}^{2}\right)$. Finally up to $18 \mathrm{~mm}$ in y-direction could be resolved for the tomographic particle image reconstruction for the both measurement volumes.

The synchronization with the flow disturbance pulsed event $(\sim 1 \mathrm{~Hz})$, the laser pulses and the camera recording is achieved using a highly accurate signal generator and a Programmable Timing Unit (PTU version 9) from LaVision, the scheme is reported in Fig. 5. By using inverted signals of $5 \mathrm{kHz}$ TTL-signals from a signal generator for the camera triggering the laser pulses were located exactly in the middle of each exposure. For each burst a set of 300 exposures was recorded. For the tripped BL case $4 \mathrm{kHz}$ framing and laser pulse rate was chosen and 1,000 images were captured for each run.

\subsubsection{Seeding}

Given the critical conditions for the light intensity in the measurement volume, a specific seeding procedure is devised in order to increase the tracer particles scattering cross section. A seeding particle generator with an array of Laskin nozzles is used with olive oil producing droplets which were introduced downstream and recirculated achieving a uniform spatial distribution. The seeding device operating temperature was decreased to 3 degrees Celsius by a refrigerator increasing the viscosity of the olive oil,

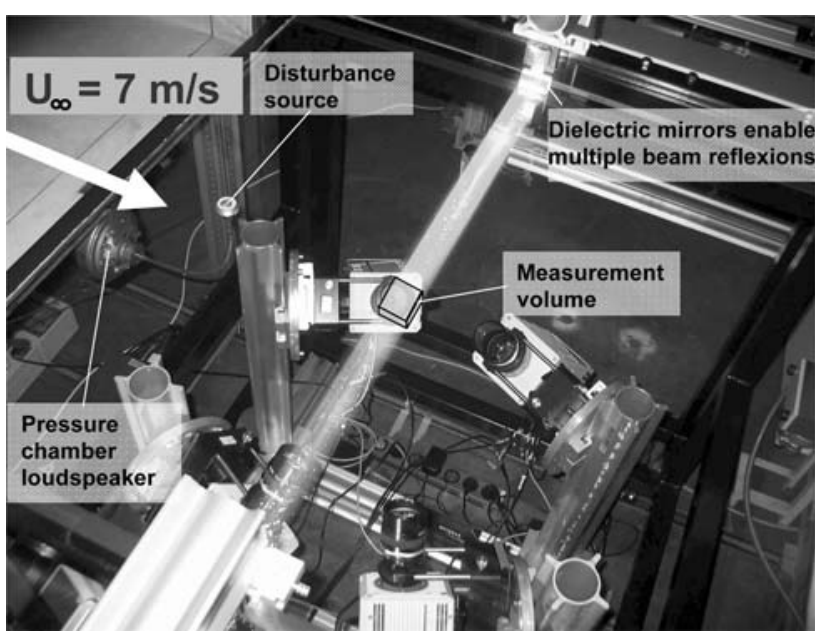

Fig. 4 Experimental set-up at a flat plate boundary layer flow at a free stream velocity of $U_{\infty}=7 \mathrm{~m} / \mathrm{s}$ consisting of four Photron APXRS CMOS cameras enabling time resolved tomographic PIV measurements of turbulent spots at the open test section of the $1 \mathrm{~m}$-wind tunnel of DLR Göttingen 


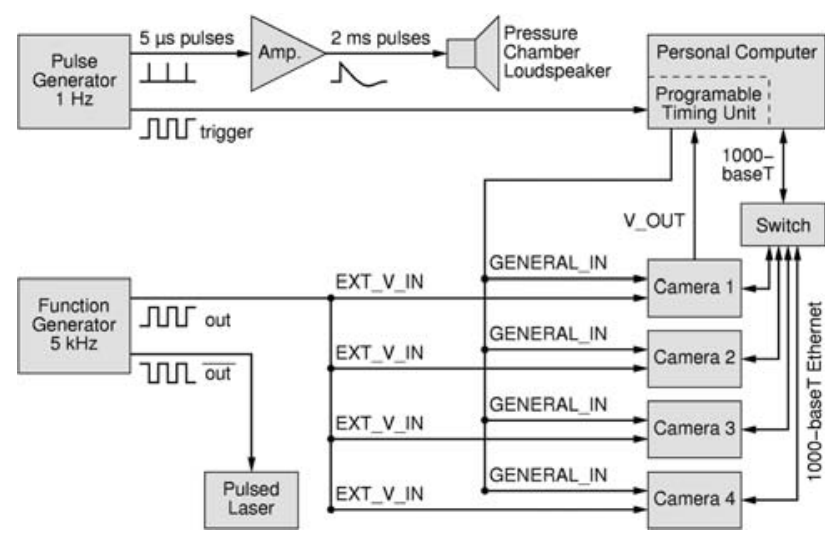

Fig. 5 Trigger scheme of the used time-resolved tomographic PIV system with synchronization by a programable timing unit

which results in tracer particles of approximately $1.5 \mu \mathrm{m}$ in diameter (Table 1).

\subsubsection{Tomographic reconstruction}

The data recording, volume calibration and calculation of the $3 \mathrm{D}-3 \mathrm{C}$ vector fields are performed using DaVis 7.3 software from LaVision. The three-dimensional particle light intensity distribution is reconstructed by means of the tomographic algorithm MART (multiplicative algebraic reconstruction technique) specialized for PIV signals returning a 3-D array of voxels representing the measurement volume where the intensity is digitally stored (Elsinga et al. 2005, 2006). The relation between image projections coordinates and the physical space in the reconstructed volume is established by a calibration procedure common to stereo PIV. Each camera records images of a 3D-calibration target at several positions in depth through the volume. A linear translation stage with stepper motor has been used in order to ensure high accuracy of the several target positions in $y$-direction. The calibration procedure returns the viewing directions and field of view by using a polynomial fitting procedure. The tomographic reconstruction relies on accurate triangulation of the views from the different cameras. In particular to successfully reconstruct the 3D light distribution in the tomogram, the accuracy requirement for the calibration is a small fraction of the particle image size. A recently developed self- calibration algorithm for tomographic PIV (Wieneke 2007) applied to the current data showed deviations of only 0.10.3 voxels of the calibration function. The tomographic reconstruction reprocessed after disparity correction, nevertheless showed an improvement in terms of noise reduction.

Before reconstruction of the tomogram, the particle image recordings require pre-processing in order to eliminate background illumination, reduce local inhomogeneities of light intensities caused by the specific illumination technique (stripes and boundary effects) and noise reduction. Sliding minimum subtraction and a Gaussian smoothing of the particle images are the applied operations. The results of these steps can be observed in Fig. 6, where on the left hand side the original particle images of all four cameras are shown. On the right hand side a slice of voxels with constant y out of the distribution of reconstructed particle images in the measurement volume is represented.

The reconstructed image distribution in the volume has been analyzed with an iterative multi-grid volume deformation scheme reaching a final $48^{3}$ voxels $(2 \times 2 \times$ $2 \mathrm{~mm}^{3}$ ) interrogation box size with $75 \%$ overlap yielding the instantaneous three-dimensional velocity volume over a grid of approximately $46 \times 24 \times 41$ for the spot case with $5 \mathrm{kHz}$ framing rate and $48 \times 24 \times 52$ measurement points for the tripped $\mathrm{BL}$ case with $4 \mathrm{kHz}$ framing rate. This field covering a box of $32 \times 18 \times 29 \mathrm{~mm}^{3}$ resp. $34 \times 18 \times$ $35 \mathrm{~mm}^{3}$ in $x-y$-z-directions for the spot and tripped turbulent $\mathrm{BL}$ respectively, while the vector plane closest to wall is at $y=2 \mathrm{~mm}$ for all cases. The possibility of reconstructing a volume of $\sim 50 \%$ thickness in $y$-direction relative to the $x$-z-extensions with a particle density of $\sim 0.03-0.1$ particles per pixel is a very promising value for this technique and was not expected on the basis of previous numerical studies (Elsinga et al. 2005).

Because nowadays the processing time for a single instantaneous velocity vector volume as described above takes about $30 \mathrm{~min}$ on a four times dual-core 64 bit PC for reconstruction and iterative volume cross-correlation only a limited number of images could be calculated up to now. Nevertheless the "4D" data with 45264 and 59048 instantaneous velocity vectors for each volume measured with 5 and $4 \mathrm{kHz}$ resp. repetition rate provide already all important information about the flow structures, which was

Table 1 Summary of experimental parameters

\begin{tabular}{llllll}
\hline Experiment & $\begin{array}{l}\text { Frame rate } \\
(\mathrm{kHz})\end{array}$ & $\begin{array}{l}\text { Active sensor } \\
\text { size }\left(\text { pixel }^{2}\right)\end{array}$ & $\begin{array}{l}\text { Number of images } \\
\text { per run }\end{array}$ & $\begin{array}{l}\text { Size of reconstructed } \\
\text { volume in } x-y-z\left(\mathrm{~mm}^{3}\right)\end{array}$ & $\begin{array}{l}\text { Vector grid } \\
\text { in } x-y-z\end{array}$ \\
\hline Turb. spot & 5 & $800 \times 768$ & 300 & $32 \times 18 \times 29$ & $46 \times 24 \times 41$ \\
Tripped TBL & 4 & $896 \times 860$ & 1,000 & $34 \times 18 \times 35$ & $48 \times 24 \times 52$ \\
\hline
\end{tabular}



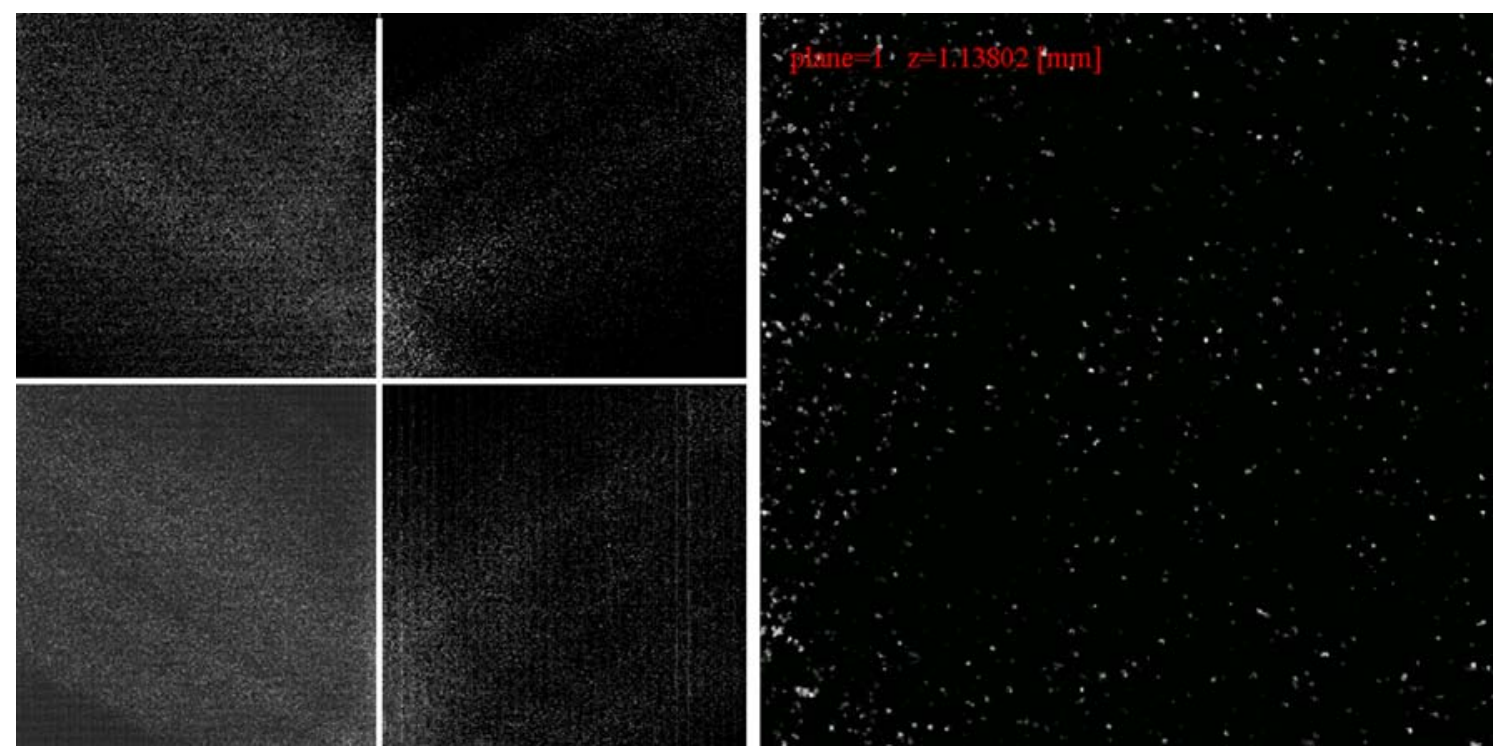

Fig. 6 Four single particle images out of the time series captured at $5 \mathrm{kHz}$ by CMOS cameras (left) and related distribution of particle images at one slice of voxels at constant $y$ within the reconstructed volume after MART algorithm applied (right)

desired often in former investigations of wall bounded turbulent flows. The number of spurious vectors was less than $3 \%$ for the shown cases and the correlation coefficient was between 25 and $75 \%$, while inside strong shear layer regions the lower coefficient value and most of the outliers appeared. For the single instantaneous velocity vector plots an uncertainty of about $\sim 3 \%$ has to be accepted, because in this feasibility study a sub-pixel accuracy of the peak detection of $\sim 0.2$ pixels is always present. Further improvements have to be done in order to enhance the accuracy from this very promising starting point.

\section{Results}

\subsection{Instantaneous 3D-3C $(t)$ velocity vector fields}

Two time steps of a sequence of the 3-D 3-C $(t)$ velocity vector volumes at the $\mathrm{LE}$ of the turbulent spot are presented in Fig. 7. In the figures the $x$-axis is directed stream-wise, the $y$-axis wall-normal and the $z$-axis span-wise. The corresponding velocity components are $V x, V y$ and $V z$. The flow structures are quantitatively visualized by the isosurfaces of the 3D swirling strength and two planes of instantaneous velocity vector fields at $y=5$ and $7 \mathrm{~mm}$. For the presentation of the 3D swirling strength iso-contour surfaces calculated with a central differencing scheme a $3 \times 3 \times 3$ vector smoothing of the results was necessary, in order to avoid displaying artificial noise. The two shown instantaneous velocity vector volumes of the same spot were measured in a volume between $y=1 \mathrm{~mm}$ and $18 \mathrm{~mm}$ and with a time-separation of $t=600 \mu \mathrm{s}$. A velocity of
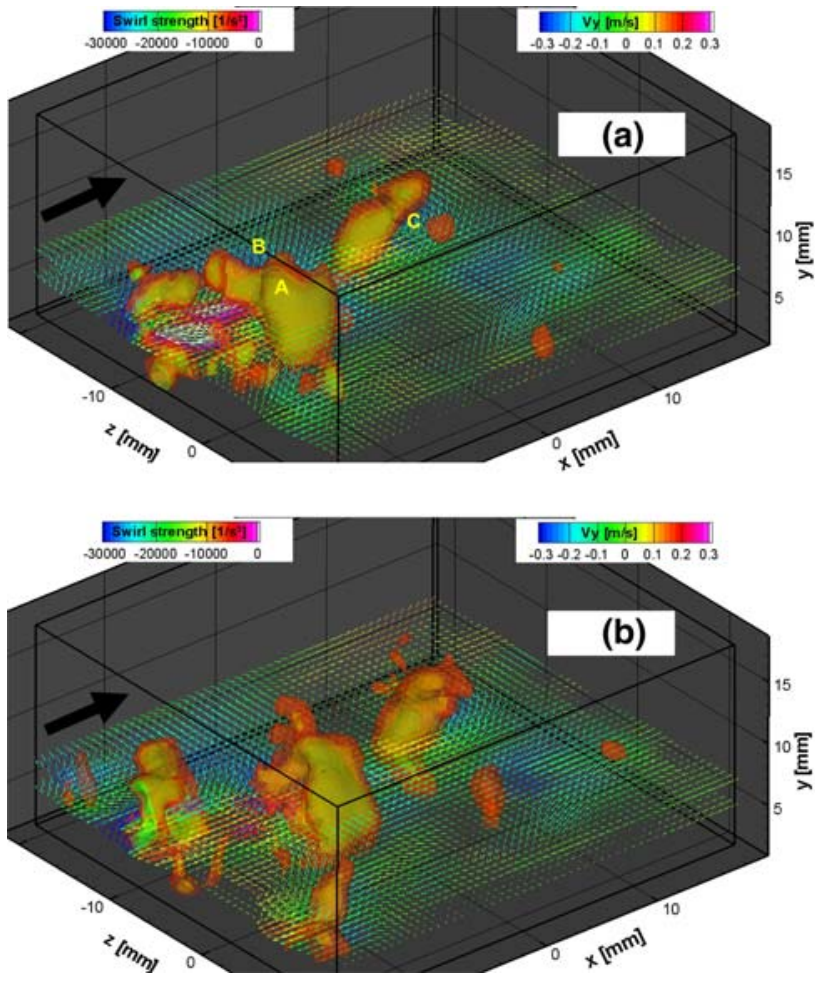

Fig. 7 Two instantaneous 3D-3C velocity vector volumes at the leading edge region of the same turbulent spot with a time separation of $t=600 \mu \mathrm{s}$. 3D swirl strength iso-surfaces and two 3C velocity vector planes at $y=5 \mathrm{~mm}$ and $7 \mathrm{~mm}$ with Q2 magnitude color coded, $\left(U_{\infty}=7 \mathrm{~m} / \mathrm{s}, V x_{\text {ref }}=V x-6.6 \mathrm{~m} / \mathrm{s}\right.$, centerline at $\left.z=-4 \mathrm{~mm}\right)$

$6.6 \mathrm{~m} / \mathrm{s}$ has been subtracted from $V x$ for visibility reasons. The first velocity vector volumes shown in Fig. 7 was captured at $T=72 \mathrm{~ms}$ and the second at $T=72.6 \mathrm{~ms}$ after 
initiation. Note that each $200 \mu$ s such a 3-D 3-C velocity vector volume has been captured. The downstream convection of hairpin-like or arch substructures can be registered. These arches of vorticity include volumes of weak Q2 events between their vortex columns visible in the positive $V y$ indicated by white vector colours at Fig. 7a). As this vector volume shows the region at the LE of the spot, the swirling maxima are located at quite high elevations $(y=5-10 \mathrm{~mm})$ confirming the overhang characteristics measured in previous investigations. Here no stream-wise swirl is visible in wall near position as proposed for the hairpin-model, which could be explained by the overhang of the "detached vortex-heads" over laminar flow. Downstream and left hand sided of the hairpin-like arch labeled ' $A$ ' a significant Q4 event region with negative $V y$ (blue vectors, B) and positive relative $V x$ velocity is located. This left hand of the swirling column located flow moves downwards and is deflected span-wise towards the low speed streak between the arch columns close to the wall. This high momentum fluid provides still sufficient energy to re-feed the process of $\mathrm{Q} 2$ production. This scenario is also visible downstream of the vortex column at downstream position $\mathrm{C}$, but with less magnitude. At the second time instance at Fig. 7b) the Q2 event magnitudes has decreased already which indicates the dissipation process of the hairpins due to their acceleration in the LE region.

Special attention will be given to the series of events leading to the rapid growth of a Q2 event, which has been observed by Schröder (2001) to occur near the TE of the spot, but could not be explained due to the limited time and spatial information in that experiment. In Fig. 8 a series of three velocity vector volumes with a time difference of $400 \mu$ s in between shall demonstrate the explosive growth of a Q2 event and related arch or hairpin-like structure at the TE of the turbulent spot. The pre-process for the rapid growing of this new turbulence producing structure is a $\mathrm{Q} 4$ event downstream of an arch vortex, visible in the related part of the precursor vector volumes (not shown here), which interacts with the downstream flow structures of the spot with much lower convection velocities. This blockage for the Q4 flow results in a deflection and change of the relative flow direction: The $\mathrm{Q} 4$ event with high velocities transforms into a slightly spanwise directed Q3 event and hitting the near-wall region upstream. The spanwise deflection is also visible in other realizations of this process. At this position only a Q2 event can help to "satisfy the continuity equation". The fluid rapidly shoots upwards against the relative flow velocity while convecting downstream and setting the precondition for the start of a new similar process. Please notice that there is no stream-wise swirl visible in the region near the wall around this Q2 event, which is in clear contradiction to classical hairpin-
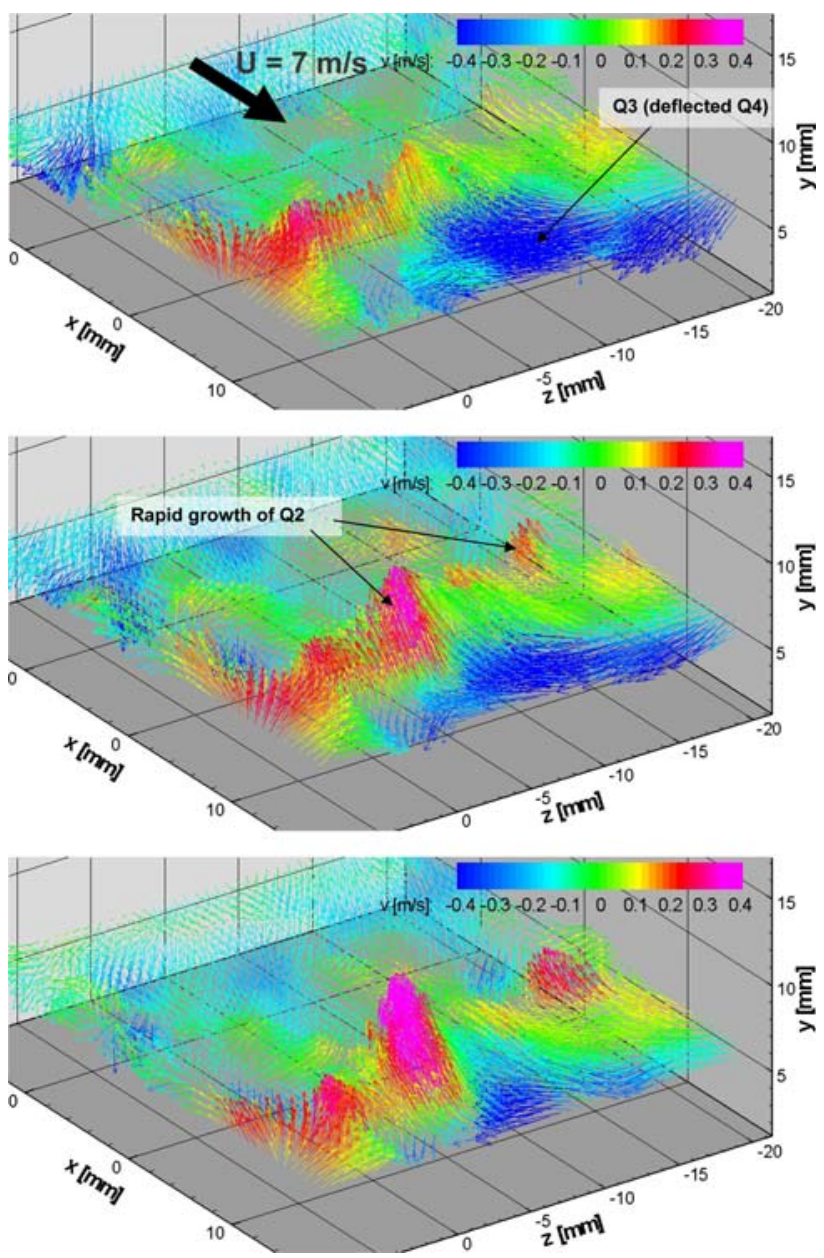

Fig. 8 Three instantaneous 3D-3C-velocity vector fields at $y=5.6$, 6.1 and $6.6 \mathrm{~mm}$ of the same turbulent spot at $T=87.4$ (top), 87.8 (centre) and $88.2 \mathrm{~ms}$ (bottom) after initial disturbance showing rapid growth of Q2 events or hairpin-like vortices at the TE of the spot $\left(U_{\infty}=7 \mathrm{~m} / \mathrm{s}, V x_{\text {ref }}=V x-6.6 \mathrm{~m} / \mathrm{s}, V y\right.$ color coded $)$

growth models (e.g. Schoppa et al. 1997). The effect of the rapid growth of Q2 events has also been shown by Schröder and Kompenhans (2004) by means of multi-plane stereo PIV, but the number of time steps per measurement plane was limited to two, so that the whole complex process could not be fully described nor understood. This scenario has been recognized also at other TE's of spots in the tomographic PIV data-set and can be found in a more complex distribution also inside the centre of the spot structure.

In Fig. 9 such a complex distribution of the instantaneous flow inside of a turbulent spot represented by three 3Cvelocity vector planes and 3D swirling strength iso-surfaces can be seen. Besides some known arch or hairpin-like vortex structures which transports Q2 events between the vortex legs indicated by purple/white vectors a high speed streak is visible in between the two most prominent span-wise separated Q2 events. Especially the hairpin-like vortex with 
Fig. 9 Instantaneous 3D-3Cvelocity vector volume with iso-surfaces of 3D swirl strength, two vector planes at $y=4.5$ and $10.0 \mathrm{~mm}$ and one at $z=-14 \mathrm{~mm}$ in the center of a turbulent spot at $T=79 \mathrm{~ms}$ $\left(U_{\infty}=7 \mathrm{~m} / \mathrm{s}\right.$,

$\left.V x_{\text {ref }}=V x-6.6 \mathrm{~m} / \mathrm{s}\right)$

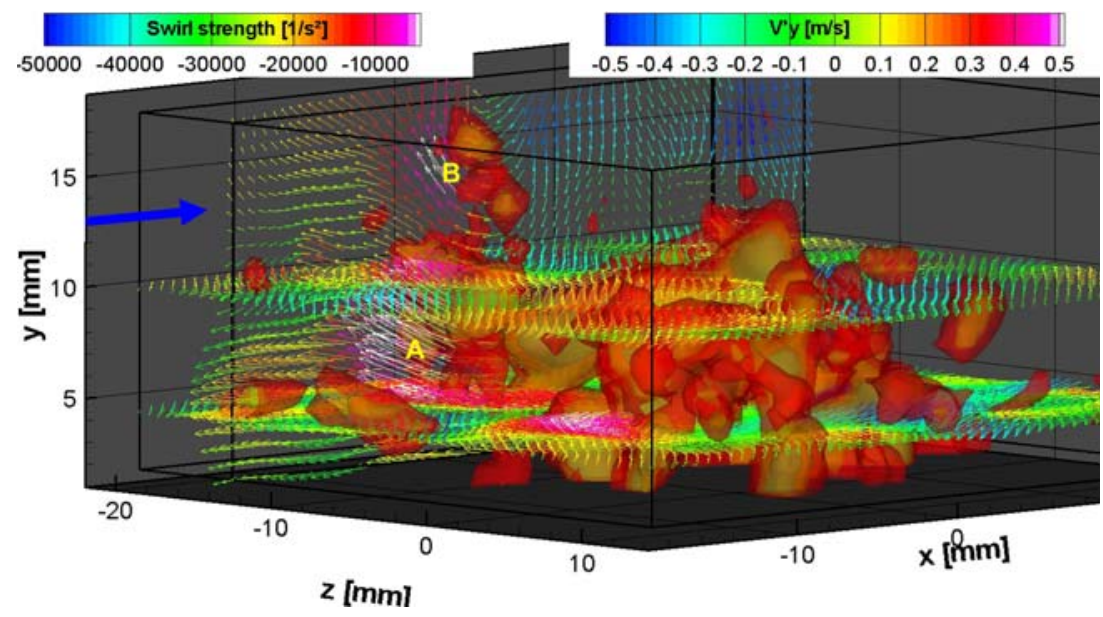

separated head at $z=-14 \mathrm{~mm}$ shows two strong Q2 events at two different heights between $y=5-8 \mathrm{~mm}$, labeled by A, and at $y=12-15 \mathrm{~mm}$, labelled by B. At the downstream positions of the single hairpin-like vortices negative $V y$ velocity can be detected. High momentum fluid is led down to the low speed streaks where $V z$ fluctuations, basically predefined by the direction possible between or simply guided by the asymmetry of the hairpin-like structures, are the reason for the interaction with low momentum fluid guiding to developments of new hairpin-like vortices. Inside the dense package of streaks and hairpin-like vortices inside the spot this leads to the staggered and streaky pattern found in previous multi-plane Stereo PIV investigations (Schröder and Kompenhans 2004). Unfortunately the whole spatiotemporal process cannot be presented within a few figures in the frame of the present paper. However, these structures will have to be analyzed in more detail in future work, although statistical approaches like space-time-correlations cannot be applied to this spot measurements reasonably on the basis of only 6 time series of 300 instantaneous 3D-PIV vector volumes representing the spot convection through the measurement volume.

In the following some results with the focus on the structures found in a wire tripped flat plate turbulent BL flow shall be presented. The average flow velocity of 500 velocity vector volumes has been determined and subtracted from each single realization. Four instantaneous velocity fluctuation vector volumes, with respect to this non-converged average profile, with a time separation of $500 \mu$ s each are represented in Fig. 10 by 3D iso-vorticity contour surfaces and two $3 \mathrm{C}$-velocity vector fields in two $x-y$-planes with $2.5 \mathrm{~mm}$ distance. Again we find the representative arch structure with negative $V^{\prime} x$ velocities included, but also here no stream-wise vorticity is present

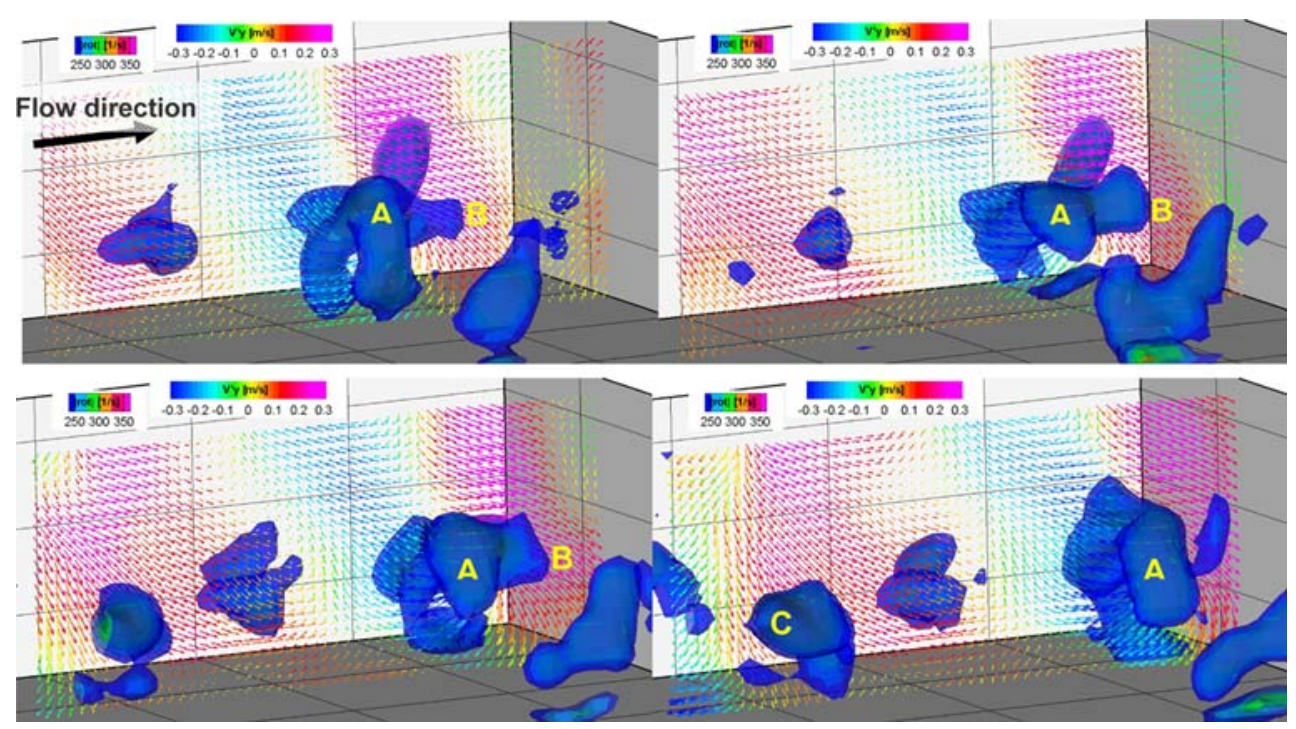

Fig. 10 Time series of instantaneous 3D iso-vorticity surfaces and two $x-y$-planes of 3C fluctuation velocity vector fields with $t=500 \mu$ s shift each in a tripped turbulent boundary layer, $V y$ color coded, $U_{\infty}=7 \mathrm{~m} / \mathrm{s}$ 
at the low speed streaks or at the feet of the arch or hairpinlike vortices. A strong Q2 event region is located directly upstream of this vortex structure labelled by A, while a Q4 event region is located directly downstream (B). Following the flow development through time the negative $V^{\prime} x$ in between the mentioned arch turns into a Q2 event and together with the deflected Q4 event upstream the flow motion results in a new Q2 event and the related vortex pair or arch structure (C) following the path of the downstream one and here almost the same velocity distribution can be found up- and downstream of this structure. This separated following of such hairpin-like structures occurs mostly not in one path of constant $\mathrm{z}$-axis position but in a laterally shifted upstream position. This behaviour might indicate that the hairpin topologies are more or less self-sustaining structures also as singular realizations. While they are convecting downstream a part of the high momentum fluid in a Q4 event downstream of such a structure is deflected as a Q3 event and reaches their own low speed streak on which they are "riding", which re-feeds the ejection of Q2. This appears especially when the hairpin-like vortex is twisted itself, as visible in Fig. 11 labelled with A, or even one-handed (B), so that the Q2 resp. Q3 event between the part closer to the wall follows the direction of the initiating laterally deflected Q4, later Q3 event, while through the upper part of the hairpin-like vortex the Q2 event is directed towards the opposite lateral direction, inducing the Q4 event on top of the structure to follow the same deflected way as the precursor flow down to the near wall position directly downstream of the hairpin-like vortex feet. But this twisting leads finally to a separation of the two "vortex legs" in stream-wise direction or in other words leads to asymmetry, confirming previous observations (Adrian et al. 2000). This process is visible exemplary in the hairpin vortex development of the right hand structure (A) in Fig. 11 from the first to the second time step shown. As a result the structure is weakened sometimes, but also re-organization occurs and a span-wise mirrored new vortex "leg" is induced (see same structure in Fig. 11 last time step shown) and finally less often a package of hairpins following each other on a wiggling low speed streak is observed as a result of this process as described in Ganapathisubramani et al. 2003. The development of the asymmetry of the new hairpin-like structure is more or less an effect of the asymmetry of the precursor. Asymmetric and/or twisted hairpin-like vortices seem to be more effective in terms of the production of new coherent flow structures than for the symmetric case. A mutual interaction of hairpin-like structures in the turbulent $\mathrm{BL}$ is essential for the near wall $V^{\prime} z$ fluctuations, which enables the creation of new (asymmetric) hairpin-like vortices at the low speed streaks. Also the ideal symmetric hairpinlike vortex structure is visible (C). A multiple vortex head

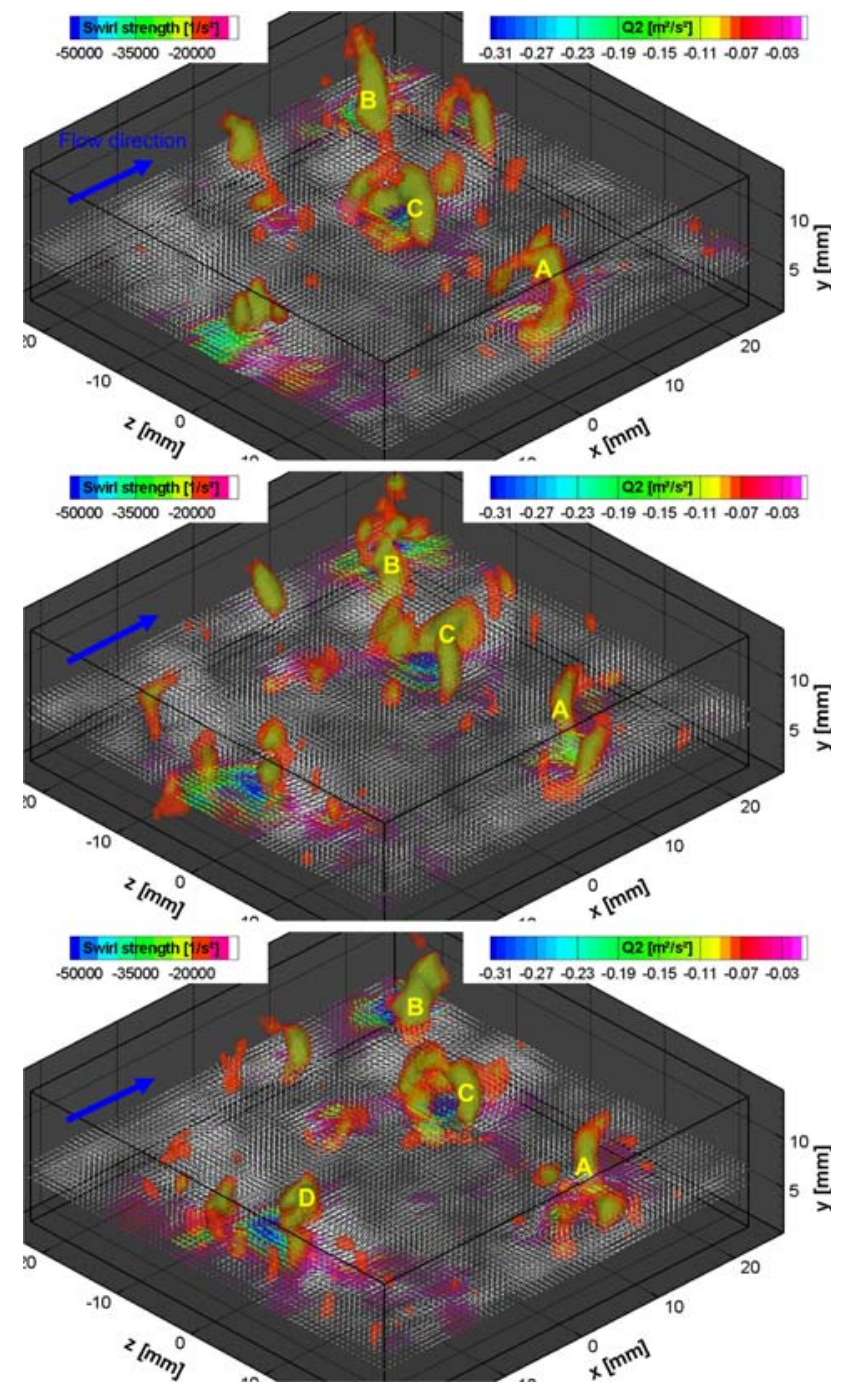

Fig. 11 Instantaneous $3 \mathrm{C}$ fluctuation velocity vector volumes of a tripped turbulent $\mathrm{BL}$ at three time steps with $500 \mu \mathrm{s}$ increment represented by iso-surfaces of swirl strength and by color coding of Q2 Reynolds stress at vectors at planes $y=6$ and $7 \mathrm{~mm}$

separation seems to occur quite frequently, while the separated head sometimes moves closer to the wall at the downstream position, (see also at D) which is known e.g. for K-type transition (Schröder 2001). Here temporal reorganisation of the arch is noticed between time step two and three of Fig. 11, which attest such structures also selfsustainability. The three instantaneous fluctuation velocity vector volumes in a time series presented in Fig. 11 should give an overview of the connection of swirling strength and Q2 events in a spatio-temporal view within a tripped turbulent BL flow. The connection of 3D swirling strength and significant Q2 events is in most of the cases connected to hairpin-like vortices or stream-wise separated vortex legs.

In summation: the particular growth mechanism of Q2 events found at the TE of the spot (Fig. 8) also occurs in a 
tripped turbulent boundary layer flow (Figs. 10, 11). However, it must be emphasized that this is only one frequently observed growth mechanism in the present data-set and that proper statistical evidence is needed to establish its relevance to boundary layer turbulence, before it could provide something like a model. In general the analysis of the instantaneous velocity vector volumes confirm existing models, but on the basis of the present data-set for the first time a spatio-temporal connection of all turbulence producing events can be provided.

\section{Conclusions}

The recently developed tomographic PIV technique has been applied to time-resolved particle images for an investigation of the sub-structures inside artificially excited turbulent spots in a laminar flat plate boundary layer flow and of coherent flow structures in a tripped turbulent boundary layer flow. Four high speed CMOS cameras are imaging tracer particles which were illuminated by two high repetitive pulse lasers in a volume inside a boundary layer flow at 4 and $5 \mathrm{kHz}$ respectively. The instantaneously acquired single particle images of these cameras have been used for a three dimensional tomographic reconstruction of the light intensity distribution of the particle images in a volume of voxels virtually representing the measurement volume. Each of two subsequently acquired and reconstructed particle image distributions are cross-correlated in small interrogation volumes using iterative multi-grid schemes with volume-deformation in order to determine a time series of instantaneous 3D-3C velocity vector fields. Future data from well defined turbulent flow experiments achieved by using time-resolved tomographic PIV will provide a valuable basis for the validation of numerical codes. A close cooperation with CFD people for the comparison with the results of numerical simulations is required as time resolved tomographic PIV can provide exactly the data needed by code developers working on the improvement of e.g. turbulence models. Nowadays, this kind of data cannot be achieved otherwise. This development may be of high interest for all flow engineering techniques and industrial aerodynamics as it includes the development of design codes and tools.

The shape and role of coherent (sub-) structures for the growth and turbulent mixing of the spot was one focus of this investigation as for the first time complete 3D topologies in a time series are available. The substructures of the spot have been identified as hairpin-like vortices arising at the rear of the spot. When these structure reaching the centre of the spot region a more complex flow topology results due to interaction with neighbouring vortical structures. Because of the local disturbance source centreline symmetry is forced and therefore the spatial configuration of the substructures inside a spot is denser, but also more orderly, than for the tripped turbulent BL flow case.

Interpretation of all results of the $3 \mathrm{D}-3 \mathrm{C}(t)$ instantaneous velocity vector volumes will take some time. This data set or the results of a more well defined generic experiment on wall bounded turbulence will enable to assess the well known models describing average coherent structures. As one result a model of the connection of the turbulence producing Q2- and Q4-events in a spatio-temporal flow topology could be achieved. Furthermore the measurement method offers the possibility to determine the complete time dependent three-dimensional velocity gradient- tensor within the measurement volume. Conditional averaging of the $3 \mathrm{D}$ velocity structure neighbouring to a given spatial event of fluid mechanical significance (like Q2) will help to construct new models of the generic coherent (sub-) structures in such flows. The fluctuation components, vorticity, Reynolds stress events and the elements of the velocity gradient tensor can be time-spacecorrelated in the whole volume. Additionally some important aspects of Lagrangian fluid dynamics of the turbulent flow can be investigated with this or suitable future data as the particles remain in the measurement volume for several (in the present data for 15-25) time steps and their trajectories could be detected by using hybrid PIV and tracking algorithms. A combined topological and Lagrangian view of the detailed flow and momentum exchange mechanisms will combine different branches of the turbulence research community and will hopefully give rise to new models and new questions resulting in an enhancement of the understanding of the structural self-organization and the energy and momentum budgets of wall bounded turbulent flows in the near future.

\section{References}

Adrian RJ, Meinhardt CD, Tomkins CD (2000) Vortex organization in the outer region of the turbulent boundary layer. J Fluid Mech 422:1-54

Cantwell B, Coles D, Dimotakis P (1977) Structure and entrainment in the plane of symmetry of a turbulent spot. J Fluid Mech 87:641-672

Coles D, Savas O (1980) Interaction of regular patterns of turbulent spots in a laminar boundary layer. In: Fasel EA, Lam.-Turb. transition, IUTAM symposium, , pp 277-288

Doorne van CWH, Hof B, Lindken RH, Westerweel J, Dierksheide U (2005) Time resolved stereoscopic PIV in pipe flow. Visualizing 3D flow structures. In: Proceedings of 5th international symposium on particle image velocimetry, Busan, Korea, 22-24 September 2003

Elsinga GE, Wieneke B, Scarano F, van Oudheusden BW (2005) Assessment of tomo-PIV for three-dimensional flows. In: Proceedings of 6th international symposium on particle image velocimetry Pasadena, California, USA, 21-23 September 2005 
Elsinga GE, Scarano F, Wieneke B, van Oudheusden BW (2006) Tomographic particle image velocimetry, experiments of fluids, 41(6), pp 933-947

Elsinga GE., Kuik DJ, Oudheusden BW, Scarano F (2007) Investigation of the three-dimensional coherent structures in a turbulent boundary layer with tomographic-PIV, AIAA 2007-1305; 45th AIAA aerospace sciences meeting and exhibition, 8-11 January 2007, Reno, Nevada

Emmons HW (1951) The laminar-turbulent transition in a boundary layer Part I. J Aeronaut Sci 18:490-498

Gad-el-Hak M, Blackwelder RF, Riley JJ (1981) On the growth of turbulent regions in laminar boundary layers. J Fluid Mech 110:73-95

Ganapathisubramani B, Longmire EK, Marusic I (2003) Characteristics of vortex packets in turbulent boundary layers. J Fluid Mech 478:35-46

Gostelow JP, Melwani N, Walker GJ (1996) Effects of streamwise pressure gradient on turbulent spot. ASME $\mathrm{J}$ Turbomach 118:737-743

Kähler CJ (2004) The significance of coherent flow structures for the turbulent mixing in wall-bounded flows. Dissertation, DLR Forschungsbericht 2004-24, ISSN 1434-8454

Matsui T (1980) Visualization of turbulent spots in the boundary layer along a flat plate in a water flow. In: Fasel EA (ed) Laminarturbulent transition, IUTAM symposium, pp 289-296

Meinhart CD (1994) Investigation of turbulent boundary-layer structure using particle-image velocimetry. Thesis, University of Illinois at Urbana-Champaign

Robinson SK (1991) The kinematics of turbulent boundary layer structure. NASA Technical Memorandum, 103859

Sabatino DR, Smith CR (2002) Simultaneous velocity-surface heat transfer behavior of turbulent spots. Exp Fluids 33:13-21

Sankaran R, Sokolov M, Antonia RA (1987) Substructures in a turbulent spot. J Fluid Mech 197:389-414
Schoppa W, Hussain F (1997) Genesis and dynamics of coherent structures in near-wall turbulence. In: Panton $\mathrm{R}$ (ed) Selfsustaining mechanisms of wall turbulence. Comput Mech Publ, pp 385-422

Singer BA (1996) Characteristics of a young turbulent spot. Phys Fluids 8:509-521

Schröder A (2001) Untersuchung der Strukturen von künstlich angeregten transitionellen Plattengrenzschichtströmungen mit Hilfe der Stereo und Multiplane Particle Image Velocimetry. http://webdoc.sub.gwdg.de/diss/2001/schroeder/schroeder.pdf

Schröder A, Kompenhans J (2004) Investigation of a turbulent spot using multi-plane stereo PIV. In: Experiments in fluids, selected issue, 36. Springer, Heidelberg, pp 82-90

Schröder A, Geisler R, Elsinga GE, Scarano F, Dierksheide U (2006) Investigation of a turbulent spot using time-resolved tomographic PIV. CD-Rom, Paper 1.4. In: Proceedings of 13th international symposium on applications of laser techniques to fluid mechanics, Lisbon (Portugal), 26-29 June 2006

Tomkins CD, Adrian RJ (2003) Spanwise structure and scale growth in turbulent boundary layers. J Fluid Mech 490:37-74

Tufo HM, Fischer PF, Papka ME, Blom K (1999) Numerical simulation and immersive visualization of hairpin vortices. http://www-unix.mcs.anl.gov/appliedmath/Flow/cfd.html

Wieneke B (2007) Volume self-calibration for stereo PIV and tomographic PIV, submitted for publication at proceedings of PIV'07, 11-14 September 2007, Rome

Wygnanski I, Zilberman M, Haritonidis JH (1982) On the spreading of a turbulent spot in the absence of a pressure gradient. J Fluid Mech 123:69-90

Zhou J, Adrian RJ, Balachandar S, Kendall TM (1999) Mechanisms for generating coherent packets of hairpin vortices in channel flow. J Fluid Mech 387:353-396 